\title{
THE INFECTION WITH HELICOBACTER PYLORI FAVORS THE PRESENCE OF GIARDIA LAMBLIA IN THE GASTRIC MUCOSA - CASE PRESENTATION
}

\author{
Meliț Lorena Elena ${ }^{1,3}$, Mărginean Cristina Oana ${ }^{1,3}$, Mocan Simona $^{2}$, \\ Suciu Nicoleta ${ }^{3}$, Mărginean Maria Oana ${ }^{3}$ \\ ${ }^{1}$ Pediatrics Clinic 1 Târgu Mureș \\ ${ }^{2}$ Department of Pathology Târgu Mureș \\ ${ }^{3}$ University of Medicine and Pharmacy Târgu Mureș
}

\begin{abstract}
Helicobacter pylori and Giardia lamblia are common worldwide. Helicobacter pylori is a favoring factor for the presence of Giardia lamblia in the stomach due to the neutralization of the gastric $\mathrm{pH}$ through the secretion of urease. We present the case of a 5-year-old child, with intermittent gastro-intestinal symptoms in the recent personal pathological history, admitted in the Pediatrics Clinic 1 Târgu Mureș for abdominal pain, nausea, loss of appetite and hematemesis. The superior digestive endoscopy revealed multiple hemorrhagic lesions of the gastric mucosa, and the pathological exam of the antral gastric mucosa identified the coexistence of Helicobacter pylori and Giardia lamblia. The evolution of the patient was favorable under the eradication therapy of the infection with Helicobacter pylori and the antiparasitic drugs, the control endoscopy revealing a gastric mucosa without macroscopically obvious modifications, and the pathological re-evaluation pointed out regenerative modifications of the gastric mucosa. The particularity of the case is represented by the identification of the parasite Giardia lamblia in the gastric mucosa, a physiologically acid environment, alkalinized by the urease secreted by Helicobacter pylori, therefore providing favorable conditions for the development of this parasite in a 5-year-old child, from a favorable socio-economic environment, with intermittent gastrointestinal symptoms in his recent personal pathological history.
\end{abstract}

Keywords: Helicobacter pylori, gastritis, Giardia lamblia, child

\section{INTRODUCTION}

Helicobacter pylori is a gram-negative, flagellated bacteria that colonizes the gastric mucosa leading to its inflammation, with possibility of becoming a chronic disorder, developing a peptic ulcer, or even gastric cancer (1). This bacteria causes the most frequent infection of the human being, being worldwide spread and being possible to appear at any age (2). The symptoms of this infection can include abdominal pain, vomiting, loss of appetite, but in many cases it is asymptomatic. Giardia lamblia, also known as Giardia intestinalis or Giardia duodenalis, is a flagellated protozoa, which affects mainly the children. The symptoms caused by this parasite can be represented by chronic diarrhea, abdominal pain, loss of appetite, but they can also lack in a high number of patients (3). Therefore in the case of asymptomatic patients, this infection can develop into a chronic one, the patients becoming reservoirs of the transmission. Giardia lamblia is commonly encountered in the duodenal mucosa, jejunum and ileum, being rarely noticed in the gastric mucosa or the colonic one (4). According to the data mentioned previously, we can 
notice that the 2 microorganisms present multiple common characteristics, such as increased frequency, the lack of clinical symptoms in most of the case, and even the possibility to become chronic disorders. Even though the presence of Giardia lamblia in the stomach is theoretically impossible under physiological conditions due to the fact that this parasite does not survive in the acid environment of the stomach, it seems that the infection with Helicobacter pylori is a risk factor for the development and surviving of this parasite in the stomach (5). The physiopathological mechanism that explains the favoring of this parasite presence in the gastric mucosa is the increased quantity of urease secreted by Helicobacter pylori, which leads to the increase of the gastric $\mathrm{pH}$ (4). This enzyme represents an adaptive mechanism of Helicobacter pylori through which the plasma urea secreted in the stomach is transformed into ammonium ions, neutralizing the acid environment of the stomach, and therefore protecting the bacteria from being destroyed by the latter one (2). The diagnostic methods for these 2 microorganisms can include serological test for Helicobacter pylori (the presence of the antibodies anti-Helicobacter pylori in the bloodstream), feces examinations for both, Helicobacter pylori and Giardia lamblia (antigen detection or parasitological exam). Nevertheless, the presence of Giardia lamblia in the stomach can be identified only by microscopic exam of the biopsy specimen taken through superior digestive endoscopy. The treatment consist in rigorous hygiene, antibiotics for the eradication of Helicobacter pylori and antiparasitic drugs for Giardia lamblia.

\section{CASE PRESENTATION}

We present the case of a 5 years and 2 months old male patient, admitted in the Pediatrics Clinic 1 Târgu Mureș due to the fact that he presented 6 episodes of vomiting, initially with bloody grooves, and afterwards with fresh blood, with an onset of approximately 12 hours, associating also nausea, loss of appetite and abdominal pain. The personal pathological history revealed that the patient complained intermittently of symptoms suggesting a gastrointestinal pathology (nausea, vomiting, abdominal pain), reasons for which he received treatment with Debridat and Nexium, and also that 3 weeks before the admission he presented an episode of acute airways infection for which he underwent antibiotic treatment for 7 days. The patient came from a medium socioeconomic environment, with good living conditions. The clinical exam performed at the moment of admission pointed out the following pathological elements: influenced general status, pallor, hyperemic pharynx, hypertrophic tonsils, with puss deposits, soft, elastic abdomen, painful spontaneously and at

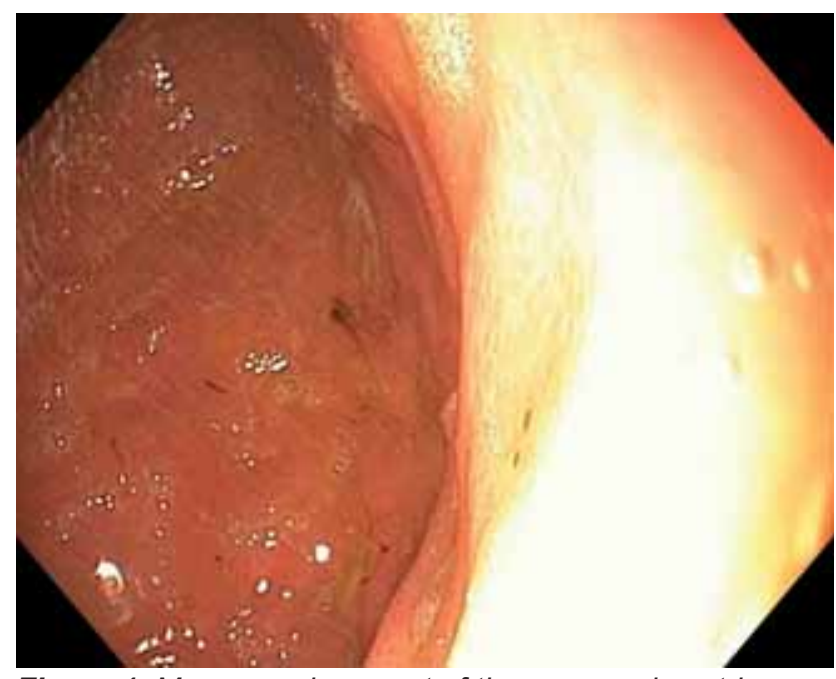

Figure 1. Macroscopic aspect of the corporeal gastric mucosa

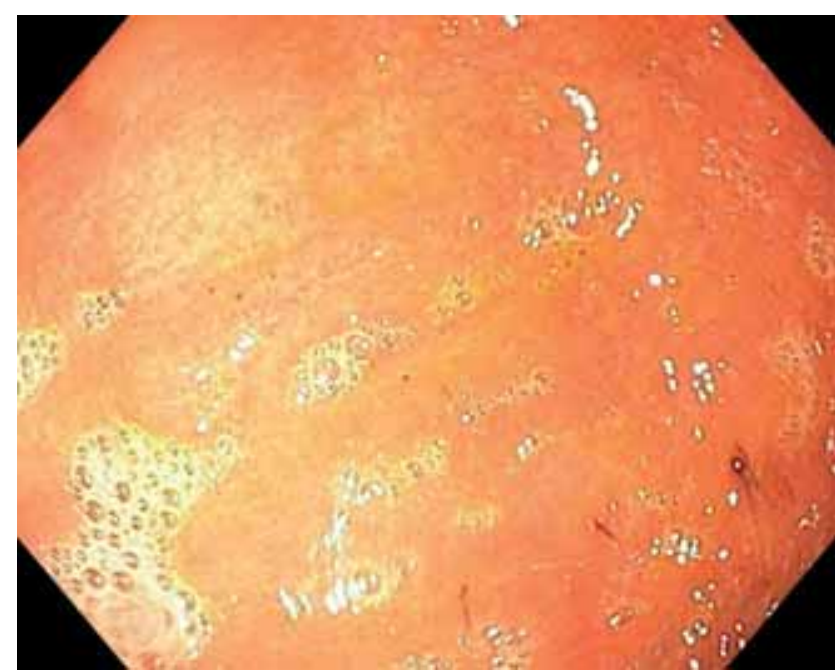

Figure 2. Macroscopic aspect of the antral gastric mucosa 
superficial and deep palpation in the epigastrium, G: $20.5 \mathrm{~kg}$.

The CBC count performed at the moment of admission revealed leukocytosis $(17,300 / \mu \mathrm{l})$ with neutrophilia (Neu: $12,200 / \mu \mathrm{l}$ ), hepatic and renal functions in normal range, as also the coagulation markers. In the second day of admission, we also performed CRP, ESR, peripheral smear and abdominal ultrasound, but without pointing out pathological elements. The macroscopic aspect of the gastric mucosa observed during the superior digestive endoscopy, performed also in the second day of admission, revealed multiple hemorrhagic lesions (Fig. 1 and 2 ). We took also biopsy specimens from the antral and corporeal mucosa in order to perform a pathological exam.

Therefore, taking under consideration the pathological elements pointed out by the pathological exam, the laboratory tests and the endoscopic aspect of the gastric mucosa, we established the following diagnoses: hemorrhagic acute gastritis and purulent acute pharyngitis, reasons for which we administered antibiotics for 5 days (Axetine 2x 1 g/day i.v.), proton pump inhibitor (Controloc $20 \mathrm{mg} /$ day) and antiemetic therapy by vein (Metoclopramid 3x $3 \mathrm{mg} /$ day i.v.). The evolution during admission was favorable, the patient being discharged after 5 of admission with the following recommendations: diet according to his actual pathology, with the avoidance of spicy food, of fats, fried aliments, and fresh fruits and vegetables, sodas, preferably to consume the aliments boiled, continuing the treatment with the proton pump inhibitor by mouth for a month.

The result of the pathological exam, at approximately one week from the moment of discharge, identified acute gastritis with Helicobacter pylori and the presence of Giardia lamblia in the gastric mucosa (Fig. 3).

Thus, the patient was recommended the eradication therapy of the infection with Helicobacter pylori by administering Amoxicillin (2x

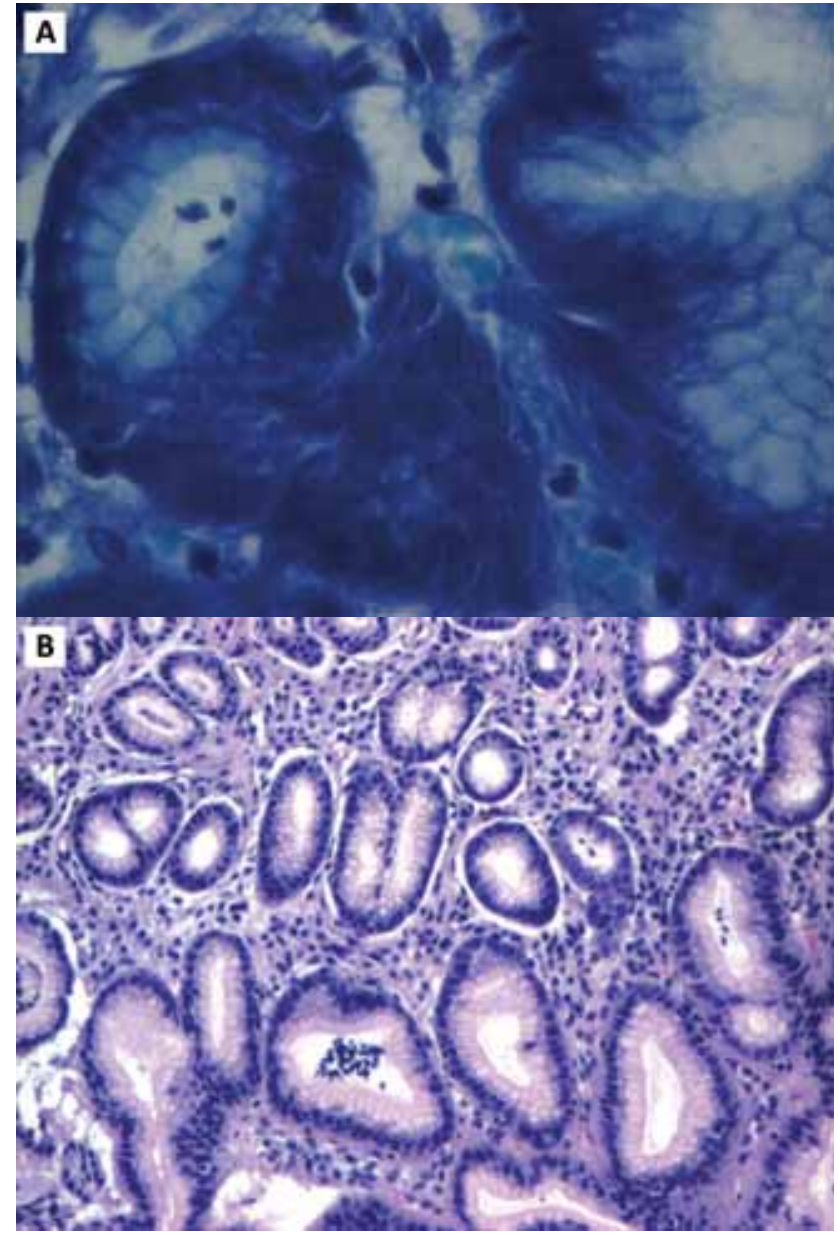

Figure 3. Histopathological aspect of the Helicobacter pylori and Giardia lamblia in the gastric mucosa ( $A$ - Giemsa stain, B - Hematoxylin-eosin stain)

$500 \mathrm{mg} /$ day) and Clarithromycin (2x $150 \mathrm{mg} /$ day) for 14 days, antiparasitic therapy with Albendazol (400 mg one dose, repeated after 7 days), the continuation of the treatment with proton pump inhibitor for a month, and endoscopic reevaluation after the recommended therapy. The endoscopy performed after approximately one month showed a normal macroscopic aspect of the gastric mucosa, and the pathological exam revealed regenerative modifications of the gastric mucosa.

The particularity of the case is represented by the identification of the parasite Giardia lamblia in the gastric mucosa, a physiologically acid environment, alkalinized by the urease secreted by Helicobacter pylori, therefore providing favorable conditions for the development of this parasite in a 5-year-old child, from a favorable socio-economic environment, with intermittent 
gastrointestinal symptoms in his recent personal pathological history.

\section{DISCUSSIONS}

The infection with Helicobacter pylori is spread worldwide, estimating that approximately $50 \%$ of the globe population is infected with this bacteria (2). Still, it seems that this infection depends on the socioeconomic development level, especially in case of children. Thus, in developed countries, such as United Sates, this bacteria is rarely encountered in children (2). On the other hand, in developing countries, the prevalence of this infection in children can reach percentages of $82-92 \%$ (6). The infection with Giardia lamblia is one of the most frequent in children, depending also on the socioeconomic level. Therefore, if the incidence in developed countries is between $2-5 \%$, this one can reach up to $20-30 \%$ in developing countries (7). Even though our patient came from a favorable socioeconomic environment, with good living conditions, he was diagnosed with the presence of a gastric coinfection with Helicobacter pylori and Giardia lamblia. Both microorganisms can produce gastrointestinal symptoms such as abdominal pain, loss of appetite, vomiting and diarrhea, but these infections can also be asymptomatic, with the possibility of becoming chronic. Thus, a study performed on 844 healthy children from Portugal, identified the presence of the infection with Giardia lamblia in a percentage of $6.8 \%$ of the cases (8). The same study showed the presence of Helicobacter pylori in 25 children of the 57 discovered with Giardia lamblia, by the detection of Helicobacter pylori antigen from the feces (8). Another study performed on 427 asymptomatic children from Uganda underlined that $20 \%$ of them were carriers of Giardia, identifying positive correlations between this parasite and the infection determined by Helicobacter pylori (9). In the above presented case, we established the diagnosis of coinfection by the identification of the two microorganisms at the patho- logical exam of the gastric mucosa. The chronic character of the infection with Giardia lamblia can lead to weight loss and growth retardation (10), while the one with Helicobacter pylori can determine peptic ulcer, digestive hemorrhage, or even gastric cancer (1). Wigren described a case of gastric mucosa infection with Giardia lamblia in a 56-year-old patient, with gastric resection in his personal history, that was associated with chronic active gastritis determined by Helicobacter pylori (11), similarly to our case. Another study, more recent, performed on 160 patients with chronic cholecystitis associated with chronic gastro-duodenitis, pointed out the presence of Giardia lamblia in the biopsy specimens of the stomach in $29.09+/-6.12 \%$ of the cases, and the frequency of Helicobacter pylori in the gastric mucosa was of $98.18+/-1.8 \%$ of the cases (12). Sabah underlined, on a study performed on a group of 206 adults with different gastrointestinal symptoms, an association of $51.4 \%$ between the infection with Helicobacter pylori and Giardia lamblia, while Eldash identified in a case-control study on 90 patients with abdominal recurrent pain and 90 healthy controls, an incidence of the coinfection with the 2 microorganisms of approximately $40 \%$ (13). Escobar-Pardo underlined also an association between Helicobacter pylori and Giardia lamblia on a study performed on 245 Brazilian children (14). Even though it is clearly established the fact that Helicobacter pylori represents a favoring factor for the presence of Giardia lamblia in the stomach, still there are multiple contradictory data that will be involved in further studies.

\section{CONCLUSIONS}

Giardia lamblia is a parasite rarely encountered in the gastric mucosa due to the fact that it survives only in an alkaline environment. The infection with Helicobacter pylori is a favoring factor for the presence of Giardia lamblia in the stomach by the neutralization of the gastric $\mathrm{pH}$ though the increased secreted urease. 


\section{REFERENCES}

1. Suerbaum S., Josenhans C. Helicobacter pylori evolution and phenotypic diversification in a changing host. Nat Rev Microbiol 2007;5:441-52

2. Kazemian H., Shavalipour A., Mohebi R. et al. Estimation of Parasitic Infection Prevalence in Children with Helicobacter pylori Infection in Ilam City (2012-2013). Arch Pediatr Infect Dis 2014;2(1):e15294.

3. Doğruman A., Kustimur S., Özekinci T., Balaban N., Ilhan M. The use of Enzyme Linked Immunosorbent Assay (ELISA) and Direct Fluorescent Antibody (DFA) Methods for Diagnosis of Giardia intestinalis. Turkiye Parazitol Derg 2006;30(4):275-278.

4. David T.J., William A.P. Markell and Voge's medical parasitology. 9th ed New York: Saunders Elsevier; 2006.

5. Sanad M.M., Darwish R.A., Nasr M.E., el-Gammal N.E., Emara M.W. Giardia lamblia and chronic gastritis. J Egypt Soc Parasitol 1996;26(2):481-95.

6. Alborzi A., Soltani J., Pourabbas B., Oboodi B., Haghighat M., Hayati M., et al. Prevalence of Helicobacter pylori infection in children (south of Iran). Diagn Microbiol Infect Dis 2006;54(4):259-61.

7. Oberhuber G., Kastner N., Stolte M. Giardiasis: A histologic analysis of 567 cases. Scand J Gastroenrol 1997;32(1):48-51.
8. Júlio C., Vilares A., Oleastro M. et al. Prevalence and risk factors for Giardia duodenalis infection among children: A case study in Portugal. Parasit Vectors 2012;5:22.

9. Ankarklev J., Hestvik E., Lebbad M. et al. Common Coinfections of Giardia intestinalis and Helicobacter pylori in Non-Symptomatic Ugandan Children. PLoS Negl Trop Dis. 2012;6(8):e1780.

10. Teixeira J., Heller L., Barreto M. Giardia duodenalis infection: risk factors for children living in sub-standard settlements in Brazil. Cad Saúde Pública 2007;23(6):1489-1493.

11. Widgren S., Pantet B., Voirol M. Giardia lamblia gastritis. A case report. Rev Med Suisse Romande 2001;121(2):153-6

12. Isaeva G.S., Efimova N.G. Gastrointestinal giardiasis associated with Helicobacter pylori. Eksp Klin Gastroenterol 2010;(6):30-4

13. Eldash H.H., Bekhit O.E., Algameel A.A. Impact of Helicobacter pylori-giardiasis coinfection on children with recurrent abdominal pain. J Egypt Soc Parasitol 2013;43(2):509-16.

14. Escobar-Pardo M.L., de Godoy A.P., Machado R.S., Rodrigues D., Fagundes Neto U., Kawakami E. Prevalence of Helicobacter pylori infection and intestinal parasitosis in children of the Xingu Indian Reservation. J Pediatr (Rio J) 2011;87(5):393-8. 